with the resultant $K_{\mathrm{i}}$ values ranging from moderate $(1,100 \mathrm{nmol} / \mathrm{l})$ to excellent $(4.6 \mathrm{nmol} / \mathrm{l})$. The most potent inhibitor, compound $2 \mathrm{~b}$, was selected for testing in an animal model of mania. In mice injected with an amphetaminechlorodiazepoxide combination, hyperactive movement was successfully inhibited by pretreatment with either valproate $(P<0.05)$ or compound $2 \mathrm{~b}(P<0.05)$.

It remains to be shown whether the behavioral effects of the synthesized compounds can be generalized to other models of mania, but this study demonstrates the success of a structure-based method for designing novel therapeutics.

Original article Kosikowski AP et al. (2007) Structure-based design leads to the identification of lithium mimetics that block mania-like effects in rodents: possible new GSK-3 $\beta$ therapies for bipolar disorders. J Am Chem Soc 129: 8328-8332

\section{Mandatory folic-acid fortification reduces prevalence of neural-tube defects in Canada}

Folic-acid supplementation during the periconceptive period reduces the risk of neuraltube defects in offspring. In 1998, the folic-acid fortification of certain cereal products became mandatory in Canada. Now, De Wals and colleagues report that the prevalence of neural-tube defects in the Canadian population has fallen following implementation of this regulation.

Among the 1,909,741 births (including still births and terminations because of fetal abnormalities) that occurred in seven Canadian provinces during the period 1993-2002, the researchers identified 2,446 subjects with neural-tube defects. On the basis of previously published results showing that red-cell folate levels started to rise in the Canadian population in April 1997, reaching a plateau in February 1999, the study period was divided into three phases: prefortification (before September 1997), partialfortification (October 1997 to March 2000), and full-fortification (April 2000 onwards). The prevalence of neural-tube defects markedly decreased from 1.58 per 1,000 births in the prefortification phase, to 0.86 per 1,000 births in the fullfortification phase. The magnitude of the decrease in the prevalence of spina bifida (53\%) was greater than that for anencephaly (38\%) or encephalocele (31\%). Historically, the prevalence of neuraltube defects has been higher in the eastern than in the western provinces of Canada. The researchers found that the greatest decreases in the prevalence of neural-tube defects occurred in the eastern provinces that had the highest prefortification prevalences, and as such, geographical differences in the prevalence of neural-tube defects almost disappeared after the full implementation of folic-acid fortification.

Original article De Wals P et al. (2007) Reduction in neural-tube defects after folic acid fortification in Canada. NEngl J Med 357: 135-142

\section{Statins reduce the incidence of dementia and Parkinson's disease to varying extents}

Previous studies have suggested that cardiovascular problems are major risk factors for the development of dementia, and that statins-drugs used to treat cardiovascular problems-might, therefore, reduce the incidence of dementia. Conflicting results in the literature, however, led Wolozin and colleagues to suggest that different statins might affect the incidence of dementia to different extents.

The researchers analyzed information from the decision support system of the US Veterans Affairs database on 727,128 patients taking simvastatin, 53,869 taking atorvastatin, and 54,052 taking lovastatin. All patients were over 64 years of age. Cox proportional hazard models were used to investigate the associations of these different agents with dementia.

The results showed that, after adjustment for factors associated with dementia or Parkinson's disease, including age, known risk factors, and Charlson comorbidity index score, use of simvastatin was associated with a significant reduction in incident dementia and incident Parkinson's disease $(P<0.0001)$. Use of atorvastatin was associated with a modest reduction in incident dementia, which was of borderline significance after adjustment for age $(P=0.11)$, but not after adjustment for other comorbidities. Lovastatin use, on the other hand, was not associated with a reduction in dementia.

The authors concluded that further investigation is warranted to establish whether these findings are attributable to the biological actions of the drugs or to an unanticipated statistical bias in the database.

\footnotetext{
Original article Wolozin B et al. (2007) Simvastatin is associated with a reduced incidence of dementia and Parkinson's disease. BMC Med 5:20
} 